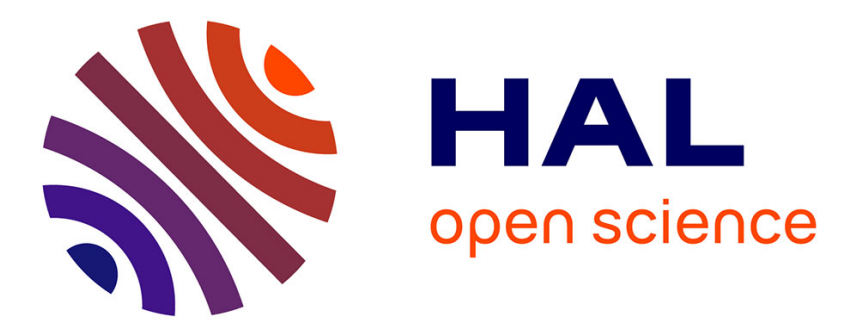

\title{
Dimorphisme sexuel et organisation sociale chez les Cercopithécinés forestiers africains
}

Annie Gautier-Hion

\section{To cite this version:}

Annie Gautier-Hion. Dimorphisme sexuel et organisation sociale chez les Cercopithécinés forestiers africains. Mammalia, 1975, 19 (3), pp.365-374. 10.1515/mamm.1975.39.3.365 . hal-01359075

HAL Id: hal-01359075

https://hal-univ-rennes1.archives-ouvertes.fr/hal-01359075

Submitted on 23 Nov 2017

HAL is a multi-disciplinary open access archive for the deposit and dissemination of scientific research documents, whether they are published or not. The documents may come from teaching and research institutions in France or abroad, or from public or private research centers.
L'archive ouverte pluridisciplinaire HAL, est destinée au dépôt et à la diffusion de documents scientifiques de niveau recherche, publiés ou non, émanant des établissements d'enseignement et de recherche français ou étrangers, des laboratoires publics ou privés. 


\title{
DIMORPHISME SEXUEL ET ORGANISATION SOCIALE CHEZ LES CERCOPITHÉCINÉS FORESTIERS AFRICAINS
}

\author{
par
}

\author{
Annie Gautier-Hion
}

\begin{abstract}
Ce travail met en évidence l'importance relative du dimorphisme sexuel chez sept espèces de Cercopithécinés forestiers qui habitent le N.-E. du Gabon. Le taux de dimorphisme sexuel est évalué pour les adultes, à partir de quatre critères : poids, longueur du dos et de la canine supérieure, superficie du sac vocal. Les espèces peuvent être classécs en trois grandes catégories. $M$. talapoin présente le dimorphisme sexuel le plus faible, suivi de Cercocebus albigena et des trois espèces arboricoles du genre Cercopithecus dont le dimorphisme sexuel est d'importance moyenne. Cercopithecus neglectus et Cercocebus galeritus au contraire ont un fort dimorphisme sexuel, tout à fait comparable à ce que l'on connait chez le Patas, le Mandrill ou l'Hamadryas.

Ainsi les variations du taux de dimorphisme sexuel chez les espèces forestières de genres voisins ou de memme genre sont tout aussi grandes que celles existant au sein de la famille entière. Une corrélation nette est mise en évidence entre grand dimorphisme sexuel - terrestrialité - et vie sociale en harems. Il apparaft que le dimorphisme sexuel dans une espèce est d'autant plus développé que le rôle joué par le mâle dans son groupe est plus différencié et exclusif.
\end{abstract}

Au fur et à mesure que de nouvelles informations nous parviennent sur l'éco-éthologie des Cercopithèques forestiers africains, on est frappé par la diversité des modes de vie décrits. La taille des bandes s'échelonne de quelques individus (Cercopithecus neglectus) à une centaine (Miopithecus talapoin), tandis que pour deux espèces d'un même genre, l'unité sociale varie de six individus chez Colobus guereza (Marler, 1969 ) à 55 chez $C$. badius (Clutton-Broke, 1972). La structure de ces diverses unités va du harem plus ou moins rigide à la société multimâles; on rencontre aussi des organisations plus complexes où les harems peuvent se mélanger temporairement : Cercocebus galeritus (Quris, 1975).

Parler "du " milieu forestier tropical conduit donc à de grandes simplifications. Parallèlement, on peut se demander quelle est l'amplitude des différences tant écologiques que morphologiques au sein des espèces d'un même genre (tels Cercopithecus ou Cercocebus), par rapport à celles relevées au sein de la famille entière. Ainsi, il est courant de dire que le dimorphisme sexuel de la taille est, de façon générale, plus important chez les singes de milieu ouvert que chez les espèces forestières,

Mammalia, t. 39, n० 3, 1975. 
pour lesquelles en outre, mâles et femelles auraient le plus souvent des canines de taille comparable. S'agit-il d'une réalité ?

En fait, notre méconnaissance de l'écologie des espèces de forêt va de pair avec une connaissance trés limitée de certaines données morphologiques et anatomiques de base. Ainsi, par exemple, la littérature ne nous apporte que peu d'informations sur le poids des Cercopithèques ou Cercocèbes adultes, et lcur croissance pondérale et staturale est quasi inconnue.

Ayant élevé des singes forestiers pendant plusieurs années, les ayant observés dans leur milieu naturel et ayant collecté de nombreux spécimens tués par les chasseurs, nous tentons, dans une note en cours, d'apporter des informations sur la croissance et la maturation de quelques espèces du Gabon. Nous voudrions ici nous attacher seulement au problème du dimorphisme sexuel et voir dans quelle mesure celui-ci varie selon les espèces et peut être mis en corrélation avec leur mode de vie. Nous établirons ensuite des comparaisons avec ce qui est connu chez les singes de milieu plus ouvert.

\section{MATÉRIEL ET MÉTHODES}

Sept espèces appartenant aux genres Miopithecus, Cercopithecus et Cercocebus font l'objet de ce travail. Toutes habitent la forêt du N.-E. du Gabon et la plupart d'entre elles ont fait l'objet d'études sur le terrain (cf. Gautier-Hion, 1971, 1973 ; Gautier-Hion et Gautier, 1974 ; Quris, 1975).

Pour mettre en évidence le dimorphisme sexuel entre animaux adultes, nous avons retenu quatre critères : le poids, la longueur du dos (mesurée de la base du crâne à la base de la queue), la longueur des canines supérieures (mesurées sur la grande courbure externe) et la superficie du sac vocal. Ce dernier critère, dont les éléments proviennent d'un travail réalisé par Gautier (1971), a été retenu en raison de l'importance majeure des annexes vocales dans l'émission des a cris forts " des mâles, cris dont le rôle dans la régulation sociale de la population a été démontré (Gautier, 1969).

Pour chaque espèce, nous comparons mâles et femelles adultes selon quatre critères. Pour chacun, la valeur moyenne obtenue pour les femelles est calculée en fonction de celle des mâles (ex. : poids moyen des mâles $=10 \mathrm{~kg}$; poids moyen des femelles $=8 \mathrm{~kg}$, soit $80 \%$.

Ainsi pour chaque critère nous obtenons un pourcentage par espèce ; les divers pourcentages sont ensuite classés par ordre décroissant, c'est-à-dire en allant du faible dimorphisme sexuel au plus fort. Il leur est alors attribué un rang :

Ex. : espèce $A: 80 \% n^{\circ} 1$ espèce $B: 70 \% n^{\circ} 2$, etc.

Les chiffres ainsi obtenus pour chaque espèce et pour chaque critère sont additionnés et fournissent un rang général classant les espèces en fonction de leur dimorphisme sexuel.

Les poids utilisés ainsi que les mesures de superficie des sacs vocaux proviennent d'animaux adultes, capturés dans leur milieu naturel. Les mensurations du dos ainsi 
que celles des canines ont été réalisées à la fois sur des animaux de la forêt et sur nos animaux d'élevage. Le critère d'adulte retenu s'appuie sur le grand développement des canines définitives, la présence des troisièmes molaires, ainsi que sur le développement normal de la peau sexuelle, l'état de gestation ou d'allaitement pour les femelles (présence du colostrum ou étirement des mamelons).

\section{RÉSULTATS}

Le tableau 1 regroupe la totalité des données, les sept espèces ayant été rangées verticalement par ordre croissant de poids moyen des mâles adultes. Notons que l'absence de mesure des annexes vocales pour Cercocebus albigena diminue pour chaque espèce, la précision de la somme des rangs d'une unité en plus ou en moins.

Si l'on considère la colonne "somme des rangs", on peut globalement établir trois catégories :

1) Somme des rangs $<6$.

Elle traduit le plus faible dimorphisme sexuel : c'est le cas du Talapoin, qui se classe en tête pour tous les critères retenus.

2) Somme des rangs $\geqslant 10$ et $\leqslant 18$.

Elle correspond à un dimorphisme sexuel moyen. Cette catégorie regroupe les trois espèces arboricoles appartenant au genre Cercopithecus. Parmi celles-ci, C. cephus et $C$. pogonias dont les mâles sont de taille très voisine, présentent également un dimorphisme sexuel très comparable ( $\Sigma$ des rangs $=13-14$ et 12-13), le cephus se classant tantôt avant pogonias (poids et longueur dorsale) tantôt après (longueur des canines, superficie du sac vocal).

De façon générale, $C$. nictitans, qui occupe les $4^{\mathrm{e}}$ et $5^{\mathrm{e}}$ rangs, présente un dimorphisme plus important que les deux espèces précédentes et pourrait peut-être constituer une classe intermédiaire.

La position de Cercocebus albigena reste incertaine du fait de l'absence de mesure des sacs vocaux. Si l'on considère toutefois les deux critères qui paraissent les meilleurs (en raison des résultats homogènes qu'ils donnent pour l'ensemble des espèces), à savoir le poids et la longueur dorsale, $C$. albigena se classe $2^{e}$, juste après le Talapoin. En outre, le $5^{\mathrm{e}}$ rang que lui attribue le critère "longueur des canines" s'explique en partie par leur morphologie. Les canines des femelles Cercocebus, à l'encontre de celles du genre Cercopithecus ne sont pas effilées comme celles des mâles, mais massives ; de ce fait, la comparaison intergénérique est délicate. Si de plus, on attribue à $\mathrm{C}$. albigena un dimorphisme sexuel maximum pour la superficie des sacs vocaux (ce que rien ne justifie particulièrement), cette espèce se maintient néanmoins dans la catégorie à dimorphisme sexuel moyen. En raison 


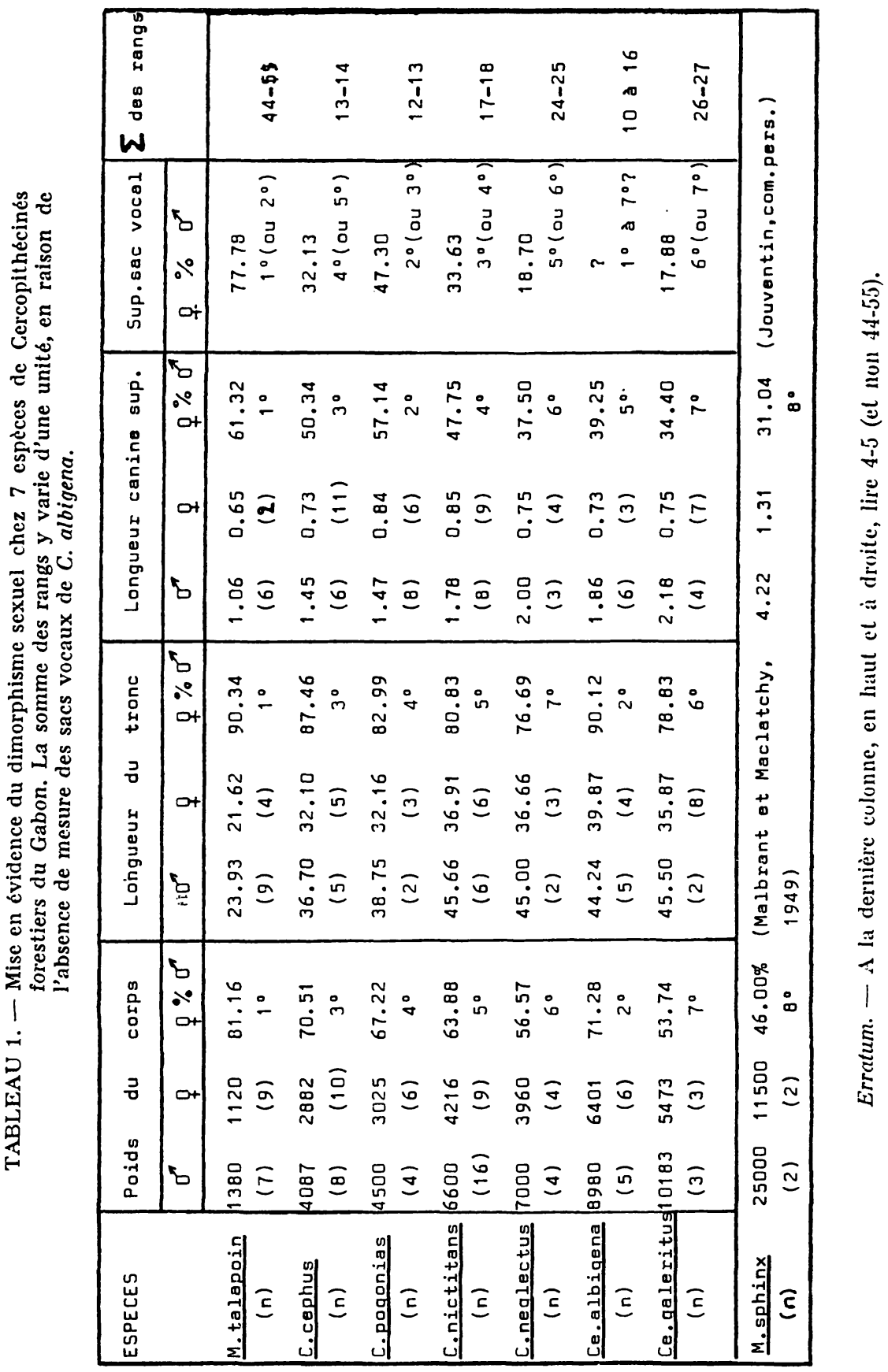


de tous ces éléments, $C$. albigena doit être considéré comme une espèce faiblement dimorphe, bien différente sous cet angle de son congénère Cercocebus galeritus et même de Cercopithecus nictitans.

3) Somme des rangs $\geqslant 24$.

Deux espèces se détachent de l'ensemble avec des rangs compris entre 24 et 27, accusant un fort dimorphisme sexuel. Il s'agit de Cercopithecus neglectus et de Cercocebus galeritus. Pour ces deux espèces, les résultats sont homogènes. $C$. neglectus se plaçant trois fois à l'avantdernier rang et Cercocebus galeritus trois fois au dernier.

\section{DISCUSSION}

Ainsi, parmi les sept espèces de Cercopithécinés habitant la forêt du N.-E. du Gabon, le dimorphisme des sexes présente de grandes variations, le poids de la femelle représentant de 54 à $81 \%$ de celui du mâle par exemple (C. galeritus; $M$. talapoin). Le rapport des poids mâles/femelles chez $C$. galeritus n'est pas éloigné de celui noté pour le Mandrill, espèce présente au N.-E. du Gabon, mais non sympatrique de la précédente. Les données de la littérature (Malbrant et Maclatchy, 1949) montrent que le poids de la femelle Mandrill est inférieur à la moitié de celui du mâle (tabl. 1). Par ailleurs, les mensurations de canines effectuées par Jouventin (com. pers.) donnent un pourcentage relatif de la longueur des canines des femelles de $31 \%$, soit une valeur à peine inférieure à celle trouvée chez Cercocebus galeritus.

Dans le genre Cercocebus, généralement considéré comme présentant un faible dimorphisme sexuel, il existe donc au moins une espèce pour laquelle le dimorphisme est extrêmement développé, selon les critères considérés. Il en est de même dans le genre Cercopithecus pour l'espèce C. neglectus. La caractéristique commune à ces deux espèces est tout d'abord qu'elles colonisent toutes deux la forêt inondée qui borde les cours d'eau et qu'elles s'y déplacent parfois au sol. Grand dimorphisme sexuel et terrestrialité se retrouvent également chez le Mandrill forestier. Mais alors que chez ce dernier le dimorphisme de taille s'accompagne d'un fort dimorphisme dans les patterns colorés de la face et dans la coloration de la peau sexuelle de la femelle, il n'en est pas de même chez Cercopithecus neglectus ni Cercocebus galeritus.

Chez $C$. galeritus, la femelle présente une peau sexuelle qui se colore cycliquement en rose violacé, mais les deux sexes ont un pelage identique et particulièrement terne. Selon Quris (1975), la coloration gris-verdâtre de cette espèce lui assure un camouflage remarquable dans le milieu dense qu'elle habite. Ainsi la sélection qui aurait conduit 
à cet important dimorphisme entre les sexes ne serait pas une sélection sexuelle au sens restreint du terme, c'est-à-dire une sélection servant à assurer à chaque sexe le maximum de chances pour l'accès au sexe opposé (sélection b de Crook, 1972, p. 264) mais plutôt le résultat de la "pression " du milieu au sens large. Vivant partiellement au sol, Cercocebus galeritus serait potentiellement plus menacé par les prédateurs ; de ce fait, selon Quris (1975) il se cantonnerait dans la forêt inondée, dont le sous-bois très dense et le sol périodiquement ịnondé et difficilement accessible constituent, très vraisemblablement, une protection pour les Mammifères qui y vivent. Pour des impératifs de défense, la sélection aurait conduit à une augmentation de la taille des mâles, sans toutefois les rendre trop visibles par des patterns colorés.

Le Mandrill au contraire, de par sa forte taille et la longueur de ses canines, est un adversaire redoutable : il habite le sous-bois plus clair de la forêt primaire et le mâle est violemment coloré.

Chez Cercopithecus neglectus, le dimorphisme sexuel apparent ne concerne que la taille ; mâles et femelles sont très colorés : bande frontale rousse, dos verdâtre, barbe et culotte blanches et la femelle ne présente pas de variations très évidentes de la peau sexuelle. Quant au scrotum bleu, il n'est visible que dans certaines positions. On peut penser que, comme chez diverses antilopes diurnes de forêt (Dubost, com. pers.) qui ont des éléments colorés voyants sous la queue ou sur la croupe, la culotte blanche du $C$. neglectus sert à la cohésion interindividuelle. Chez cette espèce, la protection est néanmoins assurée grâce à des comportements de dissimulation et d'immobilisation remarquables (Gautier-Hion, 1973), qui tendent à cacher les parties éclatantes du corps.

\section{Corrélations SOcio-Écologigues}

Le tableau 2 fait état de quelques données sur l'écologie et la structure sociale des espèces considérées. Trois conclusions essentielles s'imposent :

1) Les espèces à faible dimorphisme sexuel ont tendance à former des groupes multi-mâles, ce qui est particulièrement évident pour le Talapoin, dont les bandes sont nombreuses. Par ailleurs cette espèce n'est pas territoriale et les mâles n'y jouent pas un rôle particulièrement important dans la régulation ni la défense du groupe (absence de "cris forts " notamment). La protection est surtout assurée grâce à la coloration cryptique des animaux et à un système de diffusion rapide des alarmes vocales, associé à une grande mobilité des individus.

2) Aucune espèce à dimorphisme sexuel moyen n'habite préférentiellement la forêt inondée. Parmi les trois espèces du genre Cercopithecus 
TABLEAU 2. - Mise en évidence de certaines corrélations existant entre le taux de dimorphisme sexuel et le mode de vie.

$\mathrm{A}=$ singes arboricoles $; \mathrm{T}=$ singes partiellement terrestres

$\begin{array}{ccc}\text { Dimorphisme sexuel } & \text { Dimorphisme sexuel } & \text { Dimorphisme sexuel } \\ \text { faible } & \text { moyen } & \text { fort }\end{array}$

Forêt inondée

Forêt primaire \pm forêt secondaire
M. talapoin: A

grands groupes

multi-mâles

C. albigena : A groupes multimâles
C. cephus : A

C. pogonias: A

C. nictitans: $\mathbf{A}$ harems vrais ou a de statut "; comportement territorial chez C. nictitans
C. neglectus: $\mathrm{T}$

familles et harems

Ce. galeritus: $\mathrm{T}$ harems pouvant se joindre

M. sphinx : $\mathrm{T}$

harems réunis en grandes troupes

appartenant à cette catégorie, le dimorphisme est plus important chez C. nictitans, espèce la plus lourde et colonisant les hautes strates. Chez cette espèce, le rôle du mâle adulte, leader social du groupe, est particulièrement évident, notamment dans l'émission des "cris forts". De manière générale, les phonoréponses à caractère agressif sont extrêmement nombreuses entre mâles de bandes voisines de $C$. nictitans, rares chez $C$. pogonias et jamais observées chez $C$. cephus. En outre, dans les bandes de $C$. pogonias, il existe très souvent un second mâle adulte, ou presque adulte, susceptible d'émettre des "cris forts" d'alarme agressive en même temps que le mâle "leader ": le rôle de ce dernier semble donc moins exclusif que celui des mâles des bandes de C. nictitans. Quant aux groupes de $C$. cephus, ils ne sont pas territoriaux, leur cohésion est relativement faible et des échanges s'observent fréquemment.

Ainsi, pour ces trois espèces du même genre qui vivent en harems plus ou moins rigides et sont étroitement sympatriques puisqu'elles exploitent simultanément le même milieu au sein de troupes polyspécifiques, le dimorphisme sexuel semble d'autant plus accentué que le rôle du mâle "leader " au sein de chaque groupe est exclusif et différencié.

3) La catégorie à fort dimorphisme sexuel regroupe les trois espèces vivant partiellement au sol. C. neglectus vit en couples ou en petits harems (1 o, 2 우), habitant un petit domaine vital. Les " cris forts" des mâles sont fréquemment émis, mais certains regroupements de bandes voisines peuvent s'opérer (obs. pers.). 
Cercocebus galeritus vit en harems comprenant de 10 à 15 individus dont les domaines vitaux respectifs sont largement superposés; ces harems peuvent se joindre occasionnellement (Quris, 1975). Enfin, Mandrillus sphinx vit en troupes plus ou moins importantes qui semblent constituées du regroupement de plusieurs harems (Jouventin, com. pers.).

Nos données sur ces singes forestiers viennent donc renforcer la corrélation mise en évidence par Crook (1972) pour les singes de savane, entre terrestrialité, vie en harem et dimorphisme sexuel important. Il paraît probable que d'autres corrélations pourront être établies lorsque nous posséderons des données morphologiques détaillées, relatives non plus aux "genres ", mais aux espèces d'un même genre. Ainsi peut-être mettra-t-on en évidence dans le genre Colobus, si variable dans ses structures sociales, l'existence d'une espèce à dimorphisme sexuel plus prononcé, comme c'est le cas pour au moins une espèce des genres Cercopithecus et Cercocebus mais aussi du genre Presbytis (P. entellus, Napier et Napier, 1967, cité par Crook).

\section{Comparaison avec d'autres Cercopithécidés}

Dans son travail sur les annexes laryngées, Gautier (1971) faisait remarquer que les annexes des mâles de certains Cercopithèques étaient tout aussi développées, d'un point de vue relatif, que celles dites "hypertrophiées " de l'orang-outan par exemple. Cette même constatation peut être faite pour la taille des canines. Il est fréquent de dire que les Babouins et les Macaques sont mieux armés que les singes forestiers (à l'exception du Mandrill). Il faut cependant remarquer qu'un Talapoin de $1,3 \mathrm{~kg}$, qui possède des canines de $1,06 \mathrm{~cm}$, est proportionnellement mieux armé qu'un Mandrill de $25 \mathrm{~kg}$ dont les canines supérieures atteignent $4 \mathrm{~cm}$. Il en est de même du Cercopithecus neglectus dont le mâle possède des canines de $2 \mathrm{~cm}$ pour un poids moyen ne dépassant pas $7 \mathrm{~kg}$. Ces éléments n'ont, bien sûr, qu'une valeur relative mais tendent tout de même à montrer que beaucoup de lieux communs permettant de distinguer singes de milieu ouvert et de milieu fermé, ou, plus généralement, Babouins-Macaques et Cercopithèques, ne sont basés que sur peu de mesures précises.

En ne considérant que le critère de poids comme caractéristique du dimorphisme sexuel, il est possible d'établir des comparaisons avec d'autres singes, comparaisons déjà effectuées par Crook (1972) pour diverses espèces.

Parmi les espèces à faible dimorphisme sexuel, comparable à celui du Talapoin, on note des Colobes (C. satanas : poids de la femelle en \% de celui du mâle $=76 \%$ d'après Malbrant et Maclatchy, 1949) et la 
plupart des Langurs (environ 90\% d'après Napier, 1967). Les Cercopithèques (excepté $C$. neglectus au moins) s'apparentent aux Macaques (M. mulatta : 67,4\% d'après Schultz, 1933), tandis que $C$. neglectus et $C$. galeritus présentent un dimorphisme sexuel de poids tout aussi évident que celui de Papio hamadryas (59\% d'après Hill, 1970), du Patas (49\% d'après Hill, 1966 et Hall, 1968$)$ ou du Mandrill (46\% d'après Malbrant et Maclatchy, 1949), toutes trois espèces terrestres, dont l'unité sociale de base est le harem.

Quant à Cercopithecus aethiops, seule espèce du genre vivant essentiellement au sol et dans un milieu peu dense, son dimorphisme sexuel de poids est comparable à celui de $C$. pogonias et $C$. cephus (respectivement $69 \%$ d'après les données de Haddow, 1952, 67\% et $70 \%$ ), tandis que sa structure sociale est de type multi-mâle.

Parmi les Cercopithécinés au moins, il apparait donc que le taux de dimorphisme sexuel peut être tout aussi important entre espèces d'un même genre qu'entre genres différents et qu'il peut globalement être mis en corrélation avec l'importance du rôle joué par le mâle au sein de son groupe social.

\section{SUMMARY}

The sexual dimorphism of seven sympatric species of forest monkeys living in N.E. Gabon has been studied on living or fresh material. Its unequal importance in these species is evaluated on the basis of four different criteria : adult weight, dorsal length, upper canine length and surface-area of the vocal sacs.

The seven species can be divided into three groups : Cercopithecus neglectus and Cercocebus galeritus show the greatest sexual dimorphism, and Miopithecus talapoin the least, whereas Cercocebus albigena and Cercopithecus cephus, C. pogonias and C. nictitans are intermediary.

The degree of sexual dimorphism has therefore nothing to do with taxonomic relationships. To the contrary, it is obviously correlated with two behavioral attributes : terrestrial life and harem formation. The more important and exclusive the social role of the adult male within the group, the greater are morphological differences between adult males and females.

Station biologique de Paimpont, Universite de Rennes, 35380 Plélan-le-Grand.

\section{BIBLIOGRAPHIE}

Cuutron-Broke, T. H., 1974. - Activity patterns of red Colobus (Colobus badius tephroceles). Folia primat., $21: 161-188$.

Своок, J. H., 1972. - Sexual selection, dimorphism and social organization in the Primates. In: Sexual selection and the descent of man (B. Campbell, ed.), Aldine Pub. Co., Chicago.

Gautier, J. P., 1969. - Emissions sonores d'espacement et de ralliement par deux Cercopithèques arboricoles. Biol. gabon., 5 : 117-145. 
- 1971. - Etude morphologique et fonctionnelle des annexes extralaryngées des Cercopithecinae ; liaison avec les cris d'espacement. Biol. gabon., 7 : 229267.

Gautier, J. P. et A. Gautier-Hion, 1969. - Les associations polyspécifiques chez les Cercopithecidae du Gabon. La Terre et la Vie, $2: 164-201$.

Gautier-Hion, A., 1971. - L'écologie du Talapoin du Gabon. La Terre et la Vie, 25 : 427-490.

GAUtier-Hion, A., 1973. - Social and ecological features of Talapoin monkeys ; comparisons with others Cercopithecines. In : Comparative ecology and behaviour of Primates (R. P. Michael and J. H. Crook, eds.), London, Acad. Press : 148-170.

Gautier-Hion, A., et J. P. Gautier, 1974. - Les associations polyspécifiques de Cercopithèques du plateau de M'passa, Gabon. Folia primat., 22 : 134-177.

Hall, K. R. L., 1968. - Behaviour and ecology of the wild Patas monkey. In : Primates: studies in adaptation and variability (Ph. JAY, ed.), N.Y., Holt, Rinehart and Winston : 32-119.

Hill, O. W. C., 1966. - Primates, T. VI : Cercopithecoidea. Edinburgh Univ. Press.

- 1970. - Primates, T. VIII : Cynopithecinae. Edinburgh Univ. Press.

Malbrant, R. et A. Maclatchy, 1949. - Faune de l'Equateur africain français, T. II : Mammiferes. P. Lechevalier, Paris.

MARLER, P., 1969. - Colobus guereza : territoriality and a group composition. Science, $163: 93-95$.

Napier, J. R., and P. H. Napier, 1967. - A handbook of living Primates. London, Acad. Press.

Quris, R., 1975. - Ecologie et structure sociale de Cercocebus galeritus agilis dans le N.-E. du Gabon. La Terre et la Vie, sous presse.

Schultz, A. H., 1933. - Growth and development, C.H. II: The anatomy of the rhesus monkey. N.Y., Hafner Pub. Co. 